\title{
Awareness of Ocular Complications of Diabetes Among Diabetic Patients In A Tertiary Hospital In Western, Nigeria
}

\author{
Dr Bodunde O.T ${ }^{1,}$ Dr Odusan O ${ }^{2,}$ Dr Ogunsemi O ${ }^{2,}$ Dr Ajibode H. A ${ }^{1,}$ \\ Dr Raimi T.H ${ }^{3}$ \\ ${ }^{1}$ Department Of Ophthalomology Olabisi Onabanjo University Teaching Hospital \\ ${ }^{2}$ Department Of Medicine Olabisi Onabanjo University Teaching Hospital \\ ${ }^{3}$ Department Of Medicine, Ekiti State University Teaching Hospital
}

\begin{abstract}
:
Background:- Diabetes is no longer a disease of affluent and industrialized countries. It affects virtually all ocular structures and can lead to blindness. Routine eye examination is necessary for early detection of ocular involvement and prevention of blindness. Only few diabetics have regular eye examination.

Aim:- To study the awareness of ocular complications of diabetes among diabetic patients

Methodology:- A cross-sectional prospective study of 148 randomly selected diabetic patients was done. A semi structured questionnaire was administered to these patients by an interviewer who is medical personnel, not below the level of a house-officer. Informed consent was obtained from the patients before filling the questionnaires.

The information obtained include the biodata, educational level, duration of diabetes, whether they were aware that diabetes can affect other parts of the body, if yes which parts, which parts of the eye can it affect, how can it affect the eye, whether or not routine eye examination is necessary in diabetics, if yes how often, how did they learn about diabetes and how they think awareness of diabetic complications can be improved.

The results were collated and analyzed using SPSS version 13, with chi square done where necessary.

Results:- A total of 148 patients were studied. Their ages range between 21 years and 82years, with a mean of $58.53 \pm$ +10.4years. Majority were between 36-60yearrs of age. Eighty-three (56.1\%) had educational level within secondary level 40(27\%) tertiary education while 25(16.9\%)) do not have formal education. Most common co morbid factor was hypertension, found in 44.6\%. One hundred and twenty three (83.1\%) were aware that diabetes could affect other parts of the body, $41 \%$ were aware of brain affectation, $54 \%$ were aware that it can affect the kidneys, $77.7 \%$ were aware that it can affect the eyes, $39.2 \%$ blood vessels, $66.2 \%$ the legs, $40.5 \%$ the skin and $41.9 \%$ were aware that it can affect the genitals.

Of the $77.7 \%$ who were aware that it can affect the eyes, more than half (58.8\%) did not know the part of the eye that can be affected. $20.3 \%$ knew that it can affect the crystalline lens, $4.1 \%$ external eye, $13.5 \%$ the retina and $3.4 \%$ thought it can affect the combination of external eye, lens and the retina. Forty nine respondents $(33.1 \%)$ did not know that routine eye examination is necessary in diabetics, $16.2 \%$ says eye examination should be once, $18.2 \%$ twice, $18.9 \%$ thrice and $26.4 \%$ none. Only 18 (12.2\%) respondents got their information of eye complication from eye specialists.

Sixty- one respondents (41.2\%) do not know how diabetes can affect the eye, $29.7 \%$ knew that poor control is the problem, $8.8 \%$ think it is due to long duration, $7.4 \%$ believe it was a combination of poor control and long duration, $2 \%$ claim it is due to infection, $8.8 \%$ combination of infection, poor control and long duration and $0.7 \%$ erroneously believe thinking too much of DM can result in eye complication. Patients who have been diabetic for more than 10years are more aware that diabetes can affect the eye than those below 5years $(92.3 \%$ vs. $7.2 \%, p=0.001)$. Awareness of diabetic complication is better in those with tertiary education than others $(p=0.001)$.

Conclusion:- We conclude that though awareness of ocular complication of diabetes mellitus is high, the level of knowledge is low. Health Practitioners especially eye care specialist need to intensify health education through media, regular health talks in clinics to increase knowledge and increase health seeking behaviours of diabetics in the.
\end{abstract}

Key words: Awareness, Diabetes, Ocular complication, Diabetics

\section{Introduction}

Diabetes Mellitus used to be a disease of the affluent in Africa but not so any more with global increase in its prevalence ${ }^{1}$. Global prevalence in 1995 was put at 135,000,000 representing 5.4\% of world's population ${ }^{2}$. This is expected to increase to $300,000,000$ by 2023 representing about $6.3 \%$ of world's population ${ }^{2,3}$. Most of 
this increase is expected in developing countries put at approximately $80 \%$ while that of the developed countries is put at $40 \%^{3}$.

In Nigeria and world wide, diabetes mellitus is the commonest endocrine disorder. A study commissioned by the Federal Ministry of health in 1997 put the Nigerian prevalence at $2.2 \%{ }^{4}$. Fabiyi ${ }^{5}$ and Wokama ${ }^{6}$ actually reported that $1-7 \%$ of Nigerian population have diabetes.

Diabetes Mellitus is a multisystem disease which if left unattended to results in multi organ damage that affect mainly the vasculature of the eyes, kidneys, brain, heart and extremities. In the eye it can affect from the external structures of the eye to the retina. It can result in recurrent stye, chalazion, chronic glaucoma, cataract, vitreous haemorrhage, diabetic retinopathy, cranial nerve palsies and blindness. Diabetic Retinopathy is the most common cause of legal blindness in United States ${ }^{7}$. Loss of productivity and quality of life for any patient with diabetic retinopathy will lead to additional socio economic burdens on the community. Studies on the awareness of diabetic complication in Australia ${ }^{8}$ and United states ${ }^{9}$ showed that $37 \%$ and $65 \%$ of diabetics are aware of the association between Diabetes and eye disease. A high level of awareness is needed to educate diabetic patients due to the social disease and the economic implication that diabetic eye disease can produce ${ }^{10}$.

Majority of diabetic patients attend only the endocrinology clinics or general clinics without seeing other specialists who should co manage them like the ophthalmologists, nephrologists and dermatologists. This is possibly due to their lack of awareness that the disease can affect other parts of the body. Also many of the attending Physicians do not usually send them for routine eye check-up. Awareness about eye complications of diabetes plays an important role in encouraging people to seek timely eye care.

The aim of this study is to determine the level of awareness of eye complications among diabetic patients attending Olabisi Onabanjo University Teaching Hospital (OOUTH) and suggest ways by which this could be improved.

\section{Materials And Methods.}

A cross-sectional prospective study of 148 randomly selected patients attending the medical outpatient (Endocrine clinic) department of OOUTH with diabetes mellitus was done. A semi structured questionnaire was administered to these patients by an interviewer who is medical personnel, not below the level of a houseofficer. Informed consent was obtained from the patients before filling the questionnaires.

The information obtained include the biodata, educational level, duration of diabetes, whether they were aware that diabetes can affect other parts of the body, if yes which parts, which parts of the eye can it affect, how can it affect the eye, whether or not routine eye examination is necessary in diabetics, if yes how often, how did they learn about diabetes and how they think awareness of diabetic complications can be improved.

The results were collated and analyzed using SPSS version 13, with chi square done where necessary.

\section{Results}

A total of 148 patients were studied. Their ages range between 21 years and 82years, with a mean of $58.53 \pm 10.4$ years. Majority were between $36-60$ yearrs of age. There were $61(41.2 \%)$ males and $87(58.8 \%)$ females.

Eighty-three $(56.1 \%)$ had educational level within secondary level 40(27\%) tertiary education while 25(16.9 $\%)$ ) do not have formal education. Most common co morbid factor was hypertension, found in $44.6 \%$. One hundred and twenty three (83.1\%) do not smoke or drink alcohol, 11 (7.4\%) smoke and drink alcohol, and $14(9.5 \%)$ drink alcohol; $60 \%$ had positive family history of diabetes mellitus.

One hundred and twenty three $(83.1 \%)$ were aware that diabetes could affect other parts of the body, $41 \%$ were aware of brain affectation, 54\% were aware that it can affect the kidneys, $77.7 \%$ were aware that it can affect the eyes, $39.2 \%$ blood vessels, $66.2 \%$ the legs, $40.5 \%$ the skin and $41.9 \%$ were aware that it can affect the genitals.

Of the $77.7 \%$ who were aware that it can affect the eyes, more than half $(58.8 \%)$ did not know the part of the eye that can be affected. $20.3 \%$ knew that it can affect the crystalline lens, $4.1 \%$ external eye, $13.5 \%$ the retina and $3.4 \%$ thought it can affect the combination of external eye, lens and the retina. Forty nine respondents (33.1\%) did not know that routine eye examination is necessary in diabetics, $16.2 \%$ says eye examination should be once, $18.2 \%$ twice, $18.9 \%$ thrice and $26.4 \%$ none. Only 18 (12.2\%) respondents got their information of eye complication from eye specialists.

Sixty- one respondents (41.2\%) do not know how diabetes can affect the eye, $29.7 \%$ knew that poor control is the problem, $8.8 \%$ think it is due to long duration, $7.4 \%$ believe it was a combination of poor control and long duration, $2 \%$ claim it is due to infection, $8.8 \%$ combination of infection, poor control and long duration and $0.7 \%$ erroneously believe thinking too much of DM can result in eye complication. Four percent believe that 
awareness and knowledge of diabetic complications can be improved via the media, $37.2 \%$ by health talks and campaigns and $75.1 \%$ by communication with health workers and $2.7 \%$ do not know how we can improve awareness.

Patients who have been diabetic for more than 10years are more aware that diabetes can affect the eye than those below 5years $(92.3 \%$ vs. $7.2 \%, \mathrm{p}=0.001)$. Awareness of diabetic complication is better in those with tertiary education than others $(\mathrm{p}=0.001)$.

\section{Discussion}

Diabetes is a disease of middle age, thus it is not surprising that majority of the respondents were within age group 36-60years. This finding has been previously reported in other studies ${ }^{3,11}$. The fact that only $27 \%$ had tertiary education may be related to the agrarian lifestyle. These ones had better awareness of diabetic complication is related to the fact that they are more exposed, and seeks more knowledge of their health condition via various means. This is similar to the findings of Jajurusah et al ${ }^{7,12}$. This will also support the proposition that education is important in creating awareness.

The fact that more than three-quarter $(77.7 \%)$ of the respondents were aware of eye complication is similar to that reported by Jajurusah et al ${ }^{7}$. However, studies conducted in Australia ${ }^{7}$ and United states ${ }^{8}$ over a decade ago reported less awareness. This high level of awareness may actually be due to the fact that it is a hospital based study. Awareness does not necessarily translate to knowledge. Hearing about a problem is awareness but understanding the causes and treatment of a disease is knowledge. Despite the high level of awareness about eye complications in diabetes among respondents in this study, their knowledge about the parts of the eyes affected was rather low. More than half of them did not know the parts of the eye that diabetes affects. This finding is consistent with the trend reported in other countries ${ }^{13,14,15}$. Furthermore only a small number of respondents $(13.5 \%)$ knew that it can affect the retina. This is disturbing since retina affectation is one of the causes of blindness in western societies ${ }^{16}$. Also only few patients knew that long duration and poor control are factors that can predispose to eye involvement and as high as 33\% did not know that it is necessary for diabetics to have routine eye check-up .Hence the need to raise awareness and knowledge so as to increase the health seeking behavior of diabetics.

Our finding of hypertension as the most common co morbidity is not out of place since it has been reported as a risk factor to developing diabetic retinopathy ${ }^{17}$ while the most common risk factor for the development of diabetes found in this study was the positive family history. Another important finding in this study is the low impact of eye specialist as a source of information on diabetic related eye complication since only $18(12.2 \%)$ reported that they got information from eye specialists about ocular complications of diabetes. It is therefore necessary for ophthalmologists and other eye care workers are more involved health educating diabetics on the ocular complications through the media, campaigns, and health talks in their hospitals.

Regular comprehensive eye examination should be done in diabetics at least once yearly for early detection and screening for diabetic retinopathy, however once there is sign of retinopathy such individual should be examined at least twice yearly. This study showed

that we need to educate diabetics more of the necessity of this routine eye examination.

Annual visual acuity check should also be done for diabetics to screen for cataract. When visual acuity is less than $6 / 18$, they should be referred to an eye care specialist for full eye examination to rule out cataract, refractive error or glaucoma.

We conclude that more health education should be done for diabetics, so that they can visit eye care specialists regularly and routinely to increase early detection of eye involvement thereby reducing risk of blindness. Eye care Specialists should be more involved in educating these groups of people.

\section{References}

[1]. Global initiative for the eliminating of avoidable blindness. An informal consultation, World Health Organization, Geneva,1997,WHO/PBL/97.61

[2]. International Diabetes Federation,2005,http://www.idf.org(accessed Feb. 20 $\left.{ }^{\text {th }} 2005\right)$

[3]. Wild S, Roglic C, Green A, Sicrete R, King H. Global prevalence of Diabetes: Estimates for the year 2000 and projections for 2030. Diabetes Care 2004: 27:1047-1053

[4]. Akinkugbe OO\{ED\}. National Expert Committee on Non communicable Diseases (NCD) in Nigeria. Final report of National Survey .Federal Ministry of Health, Lagos. 1997:64-90

[5]. Fabiyi AK, Kolawole BA, Adeshinto O, Ikem RT. The impact of knowledge attitude, practice and belief of type two Nigerian diabetes on Drug compliance. Diabetes Int. 2002; 291:15-17

[6]. Jajurusah I, Wong PS, Tan LT, Rokiah P, Reddy S.Awareness of eye complications and prevalence of retinopathy in the first visit to eye clinic among type 2 diabetic patients. Int J Ophthalmol 2011;4(5):519-524

[7]. Wokama FS. Diabetes and hypertension in Africa, an overview. Diabetes Int. 2002;12(12):36-40

[8]. Livingsto PM, Wood CA, McCarty CA, Harper CA, Kaeffe JE, Taylor HR. Awareness of diabetic retinopathy among people who attended a diabetic retinopathy screening program. Med J Aust 1998; 169 (2):117

[9]. Merz CN, Buse JB, Tuncer D, Twillman CB. Physician attitudesand practices and patient awareness of cardiovascular complications of diabetes. J Am Coll cardiol 2002; 40(10):1877-1881. 
[10]. Four risk factors for severe visual loss in Diabetic Retinopathy. The diabetic Retinopathy Research Group.Arch.Ophthalmol 1979;97:54-655

[11]. King H, Aubert RE, Herman WH: Global burden of diabetes, 1995-2025: prevalence, numerical estimates, and projections.. Diabetes Care 1998, 21:1414-1431

[12]. Rani PK, Raman R, Subramani S, Perumal G, Kumaramanickavel G,Sharma T. Knowledge of diabetes and diabetic retinopathy among rural population in India, and the influence of knowledge of diabetic retinopathy on attitude and practice. Rural and Remote Health 2008 http://www.rrh.org.au

[13]. Saikumar SJ,Giridhar A, Mahesh G, Elias A, Bhat S. Awareness about eye diseases among diabetic patients: a survey in South India. Community eye health journal, 2007; 20(61):16-17.

[14]. Jacob K, DiptiLisa V, Thomas M, Gurvinder K, Satish T, Bhatti SM. Study on KAP of ocular complications due to diabetes among type II diabetes visiting a Tertiary Teaching Hospital. I J H,2012;24(1):

[15]. Oyenseri-Ogbomo G.O, Abokyi S,Koffuor GA, Abokyi E. Knowledge of diabetes and its associated ocular manifestations by diabetic patients: A study at Korle-Bu Teaching Hospital, Ghana. Niger Med J 2013;54(4):217-23

[16]. Bamashmus MA, Matlhaga B, Dutton GN. Causes of blindness and visual impairment in the West of Scotland. Eye. 2004;18:257261

[17]. Knudsen LL, Lervang HH, Lunddye-Christensen S, Gorst-Rasmussen A. The North Jutland County Diabetic Retinopathy Study: Population Characteristics. Br J Ophthalmol 2006; 90(11): 1404-1409

Table 1: E ducational background of Diabetic patients

\begin{tabular}{lc}
\hline Level of Education & Number (\%) \\
Primary & $0(0)$ \\
Secondary & $83(56.1)$ \\
Tertiary & $40(27)$ \\
No formal & $25(16.9)$ \\
Total & $148(100.0)$ \\
\hline
\end{tabular}

Table 2: AWARENESS OF ORGAN INVOLMENT BY DIABETICS

\begin{tabular}{lc}
\hline ORGAN & Number (\%) \\
Brain & $61(41)$ \\
Kidney & $80(54)$ \\
Eyes & $115(77.7)$ \\
Blood vessels & $58(39.2)$ \\
Legs & $98(66.2)$ \\
Skin & $60(40.5)$ \\
Genitals & $62(41.9$ \\
\hline
\end{tabular}

Table 3:Awareness of part of eye that DM can affect by Diabetics

$\begin{array}{lc}\text { Part of eye involved } & \text { Number (\%) } \\ \text { Lens } & 30(20.3) \\ \text { External eye } & 6(4.1) \\ \text { Retina } & 20(13.5) \\ & 5(3.4) \\ \text { Ext eye, retnia, lens } & \\ & 87(58.8) \\ \text { Don't know } & 148(100.0) \\ \text { Total } & \end{array}$

Table 4: Awareness of How Diabetes affects the eye by Diabetic patients

\begin{tabular}{lc}
\hline How & Number $(\%)$ \\
Don't know & $61(41.2)$ \\
Poor Control & $44(29.7)$ \\
Long Duration & $13(8.8)$ \\
Poor control/long duration & $11(7.4)$ \\
Infection & $3(2)$ \\
Infection/poor control/long duration & $13(8.8)$ \\
Thinking too much of DM & $1(0.76)$ \\
Total & $148(100.0)$ \\
\hline
\end{tabular}

